\title{
Research on Modeling of Government Debt Risk Comprehensive Evaluation Based on Multidimensional Data Mining
}

\section{Li ChaoYing ( $\sim$ lcy171141@163.com)}

Central University of Finance and Economics

\section{Wu Xiang Da}

Hubei University

Zhao En Hui

Chengdu University of Information Technology

\section{Research Article}

Keywords: Multidimensional data mining, Government debt risk, Comprehensive evaluation, Evaluation modeling, KMV model

Posted Date: September 7th, 2021

DOl: https://doi.org/10.21203/rs.3.rs-852683/v1

License: (c) (i) This work is licensed under a Creative Commons Attribution 4.0 International License. Read Full License

Version of Record: A version of this preprint was published at Soft Computing on December 1st, 2021. See the published version at https://doi.org/10.1007/s00500-021-06478-7. 


\title{
Research on Modeling of Government Debt Risk Comprehensive Evaluation Based on Multidimensional Data
}

\section{Mining}

\author{
Li ChaoYing ${ }^{1 *}$, Wu Xiang Da ${ }^{2}$, Zhao En Hui ${ }^{3}$ \\ 1. Finance and Taxation School, Central University of Finance and Economics, 102200, Beijing, China \\ 2. School of Marxism, Hubei University, 430000, Wuhan, China \\ 3. School of Management, Chengdu University of Information Technology, 610000, Chengdu, China \\ Corresponding author and email: Li ChaoYing, lcy171141@163.com
}

\begin{abstract}
In order to solve the problems of low accuracy of data mining, high relative error rate of evaluation and long time of evaluation in traditional government debt risk evaluation methods, this paper proposes a modeling method of government debt risk comprehensive evaluation based on multidimensional data mining. The MAFIA algorithm is used for multidimensional mining of government debt risk data, and K-means clustering algorithm is used for clustering processing of mined data. According to the clustering results, the KMV model is constructed, and the uncertainty factor is used to modify the model, so as to realize the comprehensive evaluation of government debt risk by using the modified KMV model. The experimental results show that the accuracy rate of government debt risk data mining is always above $91 \%$, the relative error rate of evaluation is always below $3.4 \%$, and the average evaluation time is $0.71 \mathrm{~s}$, the practical application effect is good.
\end{abstract}

Key words: Multidimensional data mining; Government debt risk; Comprehensive evaluation; Evaluation modeling; KMV model

\section{Introduction}

At present, on a global scale, the scale of government debt has become a threat to national economic development, the important factors and with the outbreak of the global financial crisis, the economic development of many countries have suffered serious impact, and some developed countries government to ease financial and economic crisis impact on the development of national economy and threat, relevant countries enacted a series of government fiscal plan, it promotes domestic demand and economic development, thus effectively avoiding the destructive impact of the financial crisis on the national and local economy [1]. However, the implementation of these government fiscal policies has also had a negative impact, resulting in a significant increase in the sources of financial risks. Plus with the rapid development of social economy and accelerating urbanization process, increase infrastructure construction project, makes the government demand for funding growth is nonlinear, which have increased the government's fiscal pressure, so the borrowing will be looking for ways to solve these problems, such as [2], therefore makes the government debt risk increases. Therefore, in order to further measure the risk of government debt, it is of great significance to study a new government debt risk evaluation method [3].

At present, for the government debt risk assessment studies have made certain progress, and have produced many excellent research results, such as reference [4] proposed a government debt risk evaluation method based on pressure-stateresponse model, this method from the present situation of local government debt as a research foundation, analysis of government debt pressure, debt, debt paying ability, and other indicators, and analyzes the logical relationship between various indicators, in order to build the relevant evaluation index system, based on the analytic hierarchy process (AHP) is used to analyse the evaluation index weight calculation, according to the weight calculation results build pressure-state-response model, in order to complete the government debt risk assessment, but the government debt risk existing in the method of data mining to solve the problem of low accuracy the practical effect is not good. Reference [5] proposed a local government debt risk evaluation method based on the central point triangle whitening weight function, the method by investigating and collecting a number of provinces and cities government financial data, and according to these data to construct the evaluation system of local government debt risk, according to the result of evaluation index weight calculation of center of building triangular whitenization weight function. The function is used to improve the traditional risk assessment model, and the 
improved model is used to evaluate the risk of local government debt. However, it is found in the practical application that this method has the problem of low relative error rate of risk assessment of local government debt, and there is a big gap with the ideal application effect. Reference [6] proposed a local government debt risk evaluation method based on factor analysis, this method will debt pressure, solvency, growth potential as the first-level indicators, in order to build the corresponding evaluation index system of local government debt risk, and by using factor analysis method to calculate the weight of each evaluation index, to achieve the debt risk assessment and early warning interval. However, this method has a certain complexity, resulting in an increase in the time of risk evaluation of local government debt.

In order to solve the problems existing in the above methods, this paper puts forward a new modeling method of government debt risk comprehensive evaluation based on multidimensional data mining, and verifies its application performance in government debt risk evaluation through experiments.

\section{Design of modeling method for comprehensive evaluation of government debt risk}

\subsection{Data collection and processing based on multidimensional data mining}

Data mining method refers to a process in which some computer methods are used to select useful data from massive data according to certain rules. In this process, the selected data have a certain potential correlation, so this process is also called data mining or data mining [7]. In order to further improve the comprehensive evaluation accuracy of government debt risk, this paper improves the data mining method and proposes a new multidimensional data mining method to achieve the relevant research objectives.

It is assumed that the standard set of government debt risk data is $R=\left\{a_{1}, a_{2}, \ldots, a_{n}\right\}$, which $a_{i}$ is the standard value of the $i$-th data source characteristic, the upper floating value is $u_{i}$, the lower floating value is $l_{i}$, in which $n$ is the total dimension of government debt risk data [8]. On this basis, the government debt risk data set is assumed to be $D=\left\{r_{1}, r_{2}, \ldots, r_{n}\right\}$, where $r_{1}=\left\{x_{1}, x_{2}, \ldots, x_{n}\right\}$ represents the data record of the $i$-th government debt risk data source and $x_{i}$ is the actual value of the characteristics of the $i$-th data source.

Combined with the above analysis, in order to have a clearer understanding of the differences between the data in the above two different data sets, it is necessary to calculate the difference between the standard value and the real value and compare the degree of change between the value and the designed floating value. The specific calculation formula is as follows:

$$
t=\left\{\begin{array}{l}
3,0.6 \leq \sigma \leq 1.0 \\
3,0.3 \leq \sigma<0.6 \\
1,0.1 \leq \sigma<0.3 \\
0,0 \leq \sigma<0.1 \\
4,-0.1 \leq \sigma<0 \\
5,-0.3 \leq \sigma<-0.1 \\
6,-0.6 \leq \sigma<-0.3 \\
7,-1.0 \leq \sigma<-0.6
\end{array}\right.
$$

Then the difference between the real value and the standard value $\sigma$ can be calculated as follows:

$$
\sigma=\frac{x-a}{u}(2)
$$

In the above formula, $a$ represents the discretization coefficient and $u$ represents the data standard value.

In combination with the above analysis, the MAFIA algorithm is used to conduct multidimensional mining of government debt risk data. In this process, depth first search is required to complete the process of generating candidate item 
set [9], and this process is expressed as a candidate item set tree. A detailed description of the generation process is shown in Figure 1.

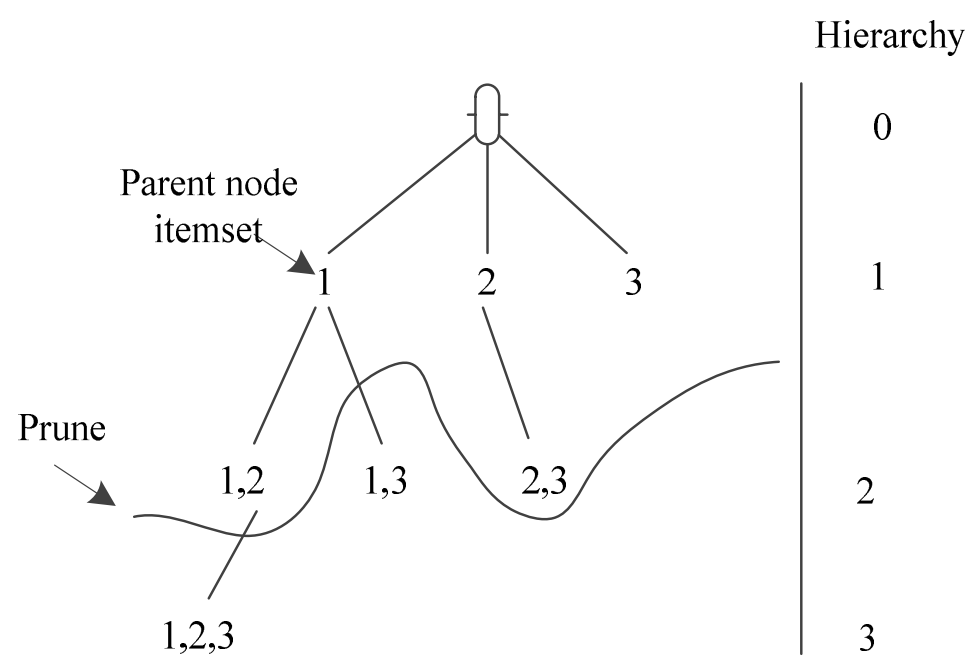

Figure 1 Candidate item set tree generation process

With each step of tree generation, a single item is expanded into a collection of multiple items. Among them, when the number of items in the item set increases gradually, the confidence level of the item set decreases accordingly. Finally, the support will be lower than the minimum support of the frequent item set. When the support is lower than the minimum support of the frequent item set, data mining will be stopped and the government debt risk data will be stored as a series of vertical bitmaps, as shown in Figure 2.

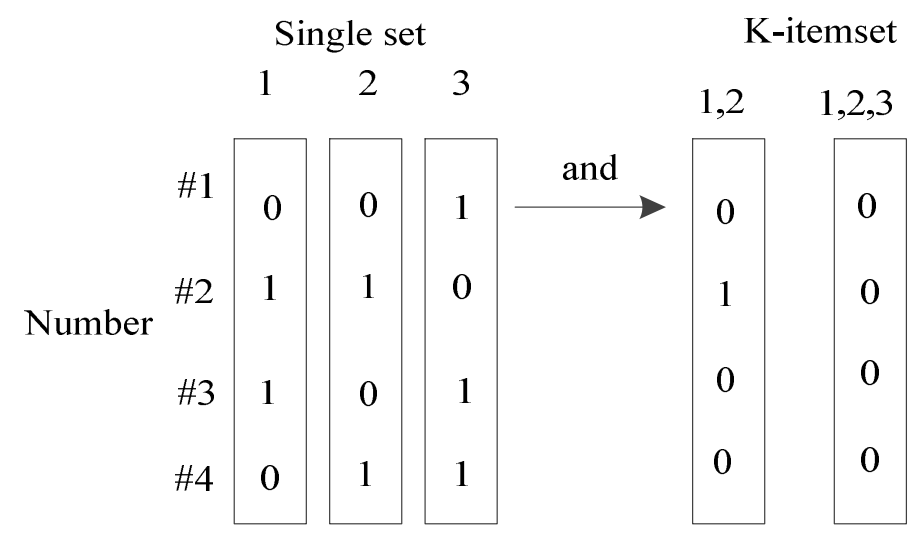

Figure 2 Concrete structure of vertical bitmap

According to the analysis of Figure 2, bits in each bitmap indicate whether there is a corresponding item set for this set of data. In the initial stage, a bitmap only corresponds to one item set. Therefore, in order to obtain the calculation result of the frequency of single item set or multinomy set, the "and" calculation between bitmaps can be achieved [10].

Combined with the above analysis, the process of applying MAFIA algorithm to obtain frequent item sets in government debt risk data is as follows:

(1) Calculate the discrete value set of the change degree of government debt risk data according to the above formula, as shown in Table 1 below.

Table 1 Set of discrete values of varying degree

\begin{tabular}{cccccc}
\hline & $\begin{array}{c}\text { Characteristics } \\
\text { of } 1\end{array}$ & $\begin{array}{c}\text { Characteristics } \\
\text { of } 2\end{array}$ & $\begin{array}{c}\text { Characteristics } \\
\text { of } 3\end{array}$ & $\ldots$ & $\begin{array}{c}\text { Characteristics } \\
\text { of } \mathrm{n}\end{array}$ \\
\hline$\# 1$ & 0 & 4 & 1 & $\ldots$ & 5 \\
$\# 2$ & 7 & 4 & 4 & $\ldots$ & $\ldots$ \\
$\ldots$ & $\ldots$ & $\ldots$ & $\ldots$ & $\ldots$ & $\ldots$ \\
\hline
\end{tabular}


(2) Change the set of discrete values in Table 1 according to the order, and there is only one number for each set.

(3) Set the minimum value of support degree. When the support degree of an item set is less than this value, a relatively frequent item set is obtained from the set of discrete values with varying degrees by combining the MAFIA algorithm. This set is the data set $F$ that is most closely related to the government debt risk [11].

After the completion of multidimensional government debt risk data mining, it is necessary to cluster the mined data. As $\mathrm{K}$-means is a partition-based clustering method, which has been widely applied in practice and received good feedback, this paper uses this algorithm to cluster government debt risk data [12]. The data clustering process is as follows:

Combined with the data mining results, the preliminary cluster set $T$ formed by DBScan was used to calculate the cluster cluster centroid set $M=\left\{m_{1}, m_{2}, \ldots, m_{h}\right\}$ according to $T$, and $k$ feature vectors $x_{1}, x_{2}, \ldots, x_{k} \in M$ were randomly selected from $M$ as the initial cluster center.

According to the above calculation formula, the relevant formula is used to call the distance from $n$-dimensional centroid to each initial cluster center $m_{i}$. The specific calculation formula is as follows:

$$
d_{(i, j)}=\sqrt{\sum_{t=1}^{n}\left(\frac{m_{i t}-x_{j t}}{s_{t}}\right)^{2}}
$$

The centroid is clustered into the cluster nearest to the point [13], and the specific calculation can be expressed by the following formula:

$$
c_{i}=\arg \min \left|d_{(i, j)}\right|(4)
$$

Combined with the above analysis, the average coordinate of all points in the clustering process is calculated, and a new clustering center is obtained according to the value. The specific formula is described as follows:

$$
x_{j}=\frac{\sum_{i=1}^{m} 1\left\{c_{i}=j\right\} m_{i}}{\sum_{i=1}^{m} 1\left\{c_{i}=j\right\}}
$$

The above process is repeated continuously. When the clustering center no longer moves in a large range and meets the convergence criterion function, the clustering is stopped and the clustering results of government debt risk data are output [14-15]. This function is described as follows:

$$
J(c, x)=\sum_{i=1}^{m}\left\|m_{i}-x_{c_{i}}\right\|^{2}(6)
$$

\subsection{Realization of government debt risk comprehensive evaluation modeling}

According to the clustering results, the KMV model is constructed, and the uncertainty factor is used to modify the model, so as to realize the comprehensive evaluation of government debt risk by using the modified KMV model. In practice, KMV model is a model derived from the option pricing formula, which can be used to calculate the default probability of the enterprise or the government, and realize the comprehensive evaluation of the debt risk of the enterprise or the government according to the result.

Because the KMV model has many advantages, such as less required parameters, high accuracy and fast speed, this paper applies the model to the comprehensive evaluation of government debt risk. Therefore, it is assumed that the government debt service revenue is a random variable that follows geometric Brownian motion and can circulate under infinite conditions, then the variable satisfies the following formula: 


$$
R_{t}=f\left(Z_{t}\right)(7)
$$

In the above formula, $R_{t}$ represents the debt service income in time $t$. At the initial time, the value is a constant, then $R_{0}=R$ exists. $Z_{t}$ represents a random variable and $f(x)$ represents a geometric Brownian motion function. Therefore, in combination with the above analysis, the following formula exists:

$$
d R_{t}=\mu R_{t} d_{t}+\sigma R_{t} d x_{t}(8)
$$

In the above formula, $d R_{t}$ represents the growth of government fiscal revenue in the period $t, d x_{t}$ represents the geometric Brownian motion growth coefficient, $\mu$ and $\sigma$ represent the growth rate and fluctuation probability of $R_{t}$ respectively.

According to ITO's theorem, the following relation exists:

$$
d=R_{0} \exp \left\{\left(\left(\mu-\frac{1}{2} \sigma^{2}\right) t+\sigma \sqrt{t} x_{t}\right)(9)\right.
$$

In general, the random variable $x_{t}$ and its logarithmic form obey normal distribution. Therefore, according to the normal distribution density function of the variable, the logarithmic form of $R_{t}$ is obtained to obtain the expected value and variance. Then, the calculation formula of the expected value is as follows:

$E\left(\ln R_{t}\right)=\ln R_{0}+\mu t-\frac{1}{2} \sigma^{2} t=\frac{1}{n-1} \sum_{i=1}^{n-1} \ln \left(R_{0} * \frac{R_{i+1}}{R_{i}}\right)=\ln R_{0}+\frac{1}{n-1} \sum_{i=1}^{n-1} \ln \left(R_{0} * \frac{R_{i+1}}{R_{i}}\right)$

The logarithmic variance expression of the debt service income variable $R_{t}$ is as follows:

$$
\operatorname{Var}\left(\ln R_{t}\right)=\sigma^{2} t=\frac{1}{n-2} \sum_{i=1}^{n-1}\left[\ln \left(\frac{R_{i+1}}{R_{i}}\right)-\frac{1}{n-1} \sum_{i=1}^{n-1} \ln \left(\frac{R_{i+1}}{R_{i}}\right)\right]^{2}
$$

Based on the above analysis and combined with the characteristic analysis results of the normal fraction function, the calculation results of $\mu$ and $\sigma$ are as follows:

$$
\begin{gathered}
\mu=\frac{\left[\frac{1}{n-1} \sum_{i=1}^{n-1} \ln \left(\frac{R_{i+1}}{R_{i}}\right)+\frac{1}{2} \sigma^{2} t\right]}{t}(12) \\
\sigma=\sqrt{\frac{\left\{\frac{1}{n-2}\left[\ln \left(\frac{R_{i+1}}{R_{i}}\right)-\frac{1}{n-1} \sum_{i=1}^{n-1} \ln \left(\frac{R_{i+1}}{R_{i}}\right)^{2}\right]\right\}}{t}}
\end{gathered}
$$

Suppose that the debt repayment date of the government is described as $T$, and the amount payable is described as $B_{T}$. When the debt repayment date of the government is $R_{T}$, the debt repayment income of the government is less than the face value of the debt payable by the government, which is $B_{T}$, then the government has the problem of bond default. The 
probability of this problem is $P$. The default probability expression and default distance of government debt can be calculated. The specific calculation formula is as follows: Suppose the government debt repayment date with description, should repay the amount with description, when the government's debt repayment date is less than it should be the face value of the debt, said the government bond default problem, the probability of the problem is, the combination of Merton model, expression and default on government debt default probability distance calculation, the specific calculation formula is as follows:

$$
\begin{gathered}
P=P\left[R_{T}<B_{T}\right]=P\left[f\left(x_{T}<B_{T}\right)\right]=P\left[x_{T}<f^{-1}\left(B_{T}\right)\right]=N\left[\frac{\left(\ln B_{T}-\ln R_{0}\right)-\left(\mu_{T}-\frac{1}{2} \sigma^{2}\right) T}{\sigma \sqrt{T}}\right] \\
D D=\left[\frac{\ln \frac{R_{T}}{B_{T}}+\left(\mu_{T}-\frac{1}{2} \sigma_{T}^{2}\right) T}{\sigma \sqrt{T}}\right](15)
\end{gathered}
$$

In general, the KMV model for the comprehensive evaluation of government debt risk can be constructed by knowing the four parameters: the government's debt repayment revenue $R_{T}$, the debt payable value $B_{T}$ and $\mu, \sigma$ at time $T$.

However, in practice, the volatility of economic development, capital and market increases the uncertainty of government debt risk. Therefore, this paper needs to optimize the model, so as to improve the comprehensive evaluation accuracy of government debt risk.

Combined with the above analysis, this paper first analyzes the expected price of European no-dividend call options under Knight uncertainty, and the specific calculation formula is as follows:

$$
C\left(S_{T}, K\right)=S_{0} e^{-\lambda \sigma T} N\left(d_{1}\right)-K e^{-r T} N\left(d_{2}\right)(16)
$$

Since government debt can also be regarded as a European call option, the traditional KMV model can be modified and optimized by using the uncertainty parameters of Knight, so as to further improve the performance of the model. Therefore, in combination with the above analysis, this paper introduces the uncertainty factor and recalculates the default probability and default distance in the case of uncertainty factor $\lambda \geq 0$, which is expressed by the following formula:

$$
\begin{gathered}
P^{\prime}=P\left[R_{T}<B_{T}\right]=P\left[f\left(x_{T}<B_{T}\right)\right]=P\left[x_{T}<f^{-1}\left(B_{T}\right)\right]=N\left[\frac{\left(\ln B_{T}-\ln R_{0}\right)-\left(\mu_{T}-\frac{1}{2} \sigma^{2}-\lambda \varsigma\right) T}{\sigma \sqrt{T}}\right] \\
D D^{\prime}=\left[\frac{\ln \frac{R_{T}}{B_{T}}+\left(\mu_{T}-\frac{1}{2} \sigma_{T}^{2}-\lambda \sigma\right) T}{\sigma \sqrt{T}}\right](18)
\end{gathered}
$$

Therefore, according to the calculation results of the new default probability and default distance, the KMV model is modified, and the comprehensive evaluation of government debt risk is realized by using this model. The detailed description of this model is as follows: 


$$
V_{E}=\frac{P^{\prime} \cdot\left(D D^{\prime} B_{T} N\left(d_{1}\right)-R_{T} N\left(d_{2}\right)+\sigma^{2}\right)}{\mu}
$$

\section{Simulation experiment design and result analysis}

\subsection{Experimental experiment design}

In order to verify the practical application effect of the modeling method of government debt risk comprehensive evaluation based on multidimensional data mining, an experimental test is carried out. In order to ensure the scientificity and reliability of the experimental results, the experiment should be carried out in the same experimental environment, as shown in Table 2.

Table 2 Experimental environment

\begin{tabular}{ccc}
\hline Runtime environment & Configuration & Parameter \\
\hline \multirow{3}{*}{ Hardware environment } & CPU & Intel(R)Core(TM)i5-9400 \\
& Frequency & $2.90 \mathrm{GHz}$ \\
& RAM & $16.0 \mathrm{~GB}$ \\
Operating system & Windows 10 \\
& Version & 18362.1082 pro \\
& Digits & $64 \mathrm{bit}$ \\
& Analog software language & APDL \\
& Simulation software & Matlab 7.0 \\
\hline
\end{tabular}

The government debt data of a local city is taken as the experimental sample data, and the experimental sample data is cleaned and repaired to reduce the experimental error and improve the accuracy of the experimental results. The evaluation method based on pressure-state-response model proposed in reference [4], the evaluation method based on central point triangle whitening weight function proposed in reference [5] and the modeling method of government debt risk comprehensive evaluation based on multidimensional data mining proposed in this paper are selected as experimental comparison methods. By comparing the accuracy of government debt risk data mining, relative error rate and time consuming of government debt risk comprehensive evaluation of different methods, the comprehensive performance of different methods is tested.

\subsection{Analysis of experimental results}

According to the above experimental scheme design, the government debt risk data mining accuracy of different methods is first compared, and the comparative results are shown in Figure 3.

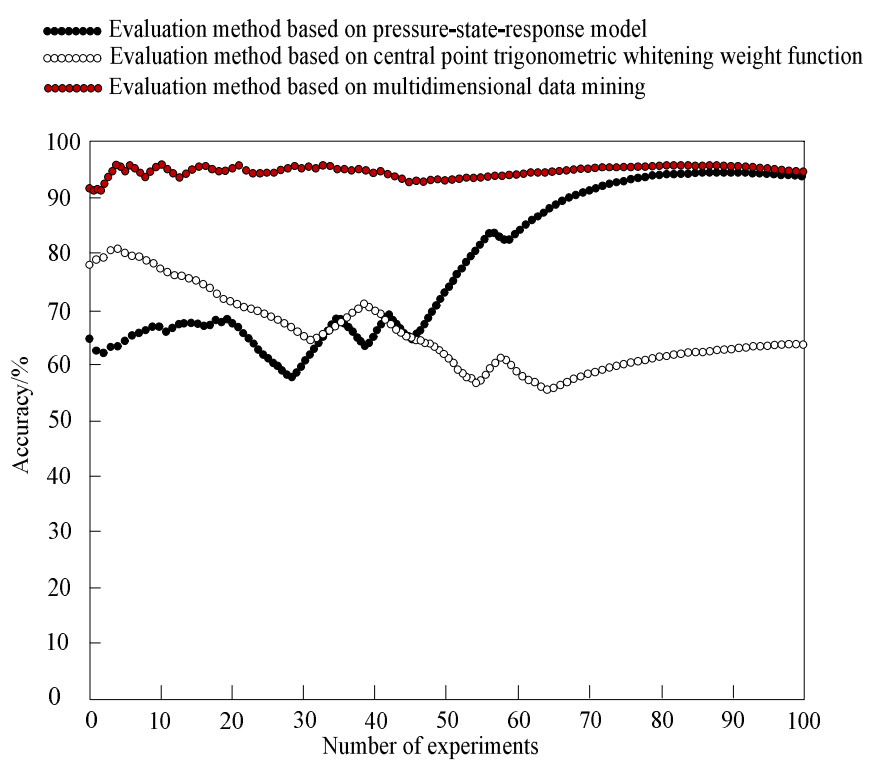

Figure 3 Comparison of data mining accuracy 
Analysis of data in Figure 3, the evaluation method based on the pressure-state-response model of data mining accuracy between $57 \%$ and $93 \%$, based on the center of the triangle whitenization weight function of the evaluation method of data mining accuracy between $55 \%$ and $82 \%$ of change, evaluation methods of data mining on the basis of multidimensional data mining accuracy is above $91 \%$ all the time, it shows that compared with the two methods, the government debt risk data mining accuracy of the proposed method is higher and the error is lower, which can lay a solid foundation for the accurate evaluation of the government debt risk in the future.

On the basis of the above, the relative error rates of the comprehensive evaluation of government debt risk of the three methods are compared, and the calculation results are shown in Figure 4.

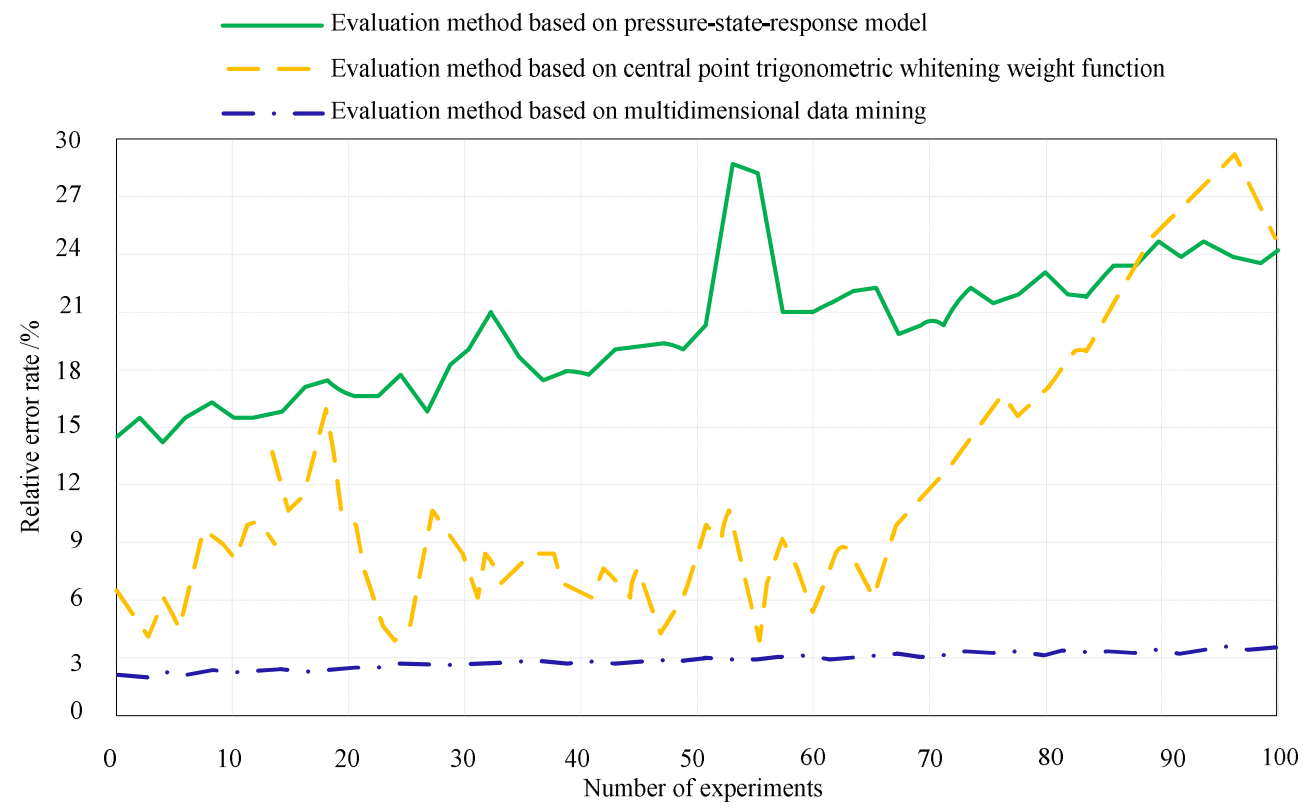

Figure 4 Comparison of relative error rates of evaluation

The analysis of data in Figure 4 shows that the evaluation relative error rate of the evaluation method based on the pressure-state-response model varies between $13.5 \%$ and $28.3 \%$, and that of the evaluation method based on the central point triangular whitening weight function varies between $4.1 \%$ and $29.1 \%$. The relative error rate of government debt risk comprehensive evaluation modeling method based on multidimensional data mining is always below $3.4 \%$, indicating that compared with the two methods, the proposed method has a lower relative error rate and higher accuracy, which can achieve accurate comprehensive evaluation of government debt risk.

Combined with the above experimental scheme design, the time consuming of the comprehensive evaluation of government debt risk of the three methods is compared. The specific results are shown in Table 3.

Table 3 Time comparison of comprehensive evaluation of Government Debt risk (unit:s)

\begin{tabular}{cccc}
\hline Number of experiment & $\begin{array}{c}\text { Evaluation method } \\
\text { based on pressure- } \\
\text { state-response model }\end{array}$ & $\begin{array}{c}\text { Evaluation method } \\
\text { based on central point } \\
\text { trigonometric } \\
\text { whitening weight } \\
\text { function }\end{array}$ & $\begin{array}{c}\text { Evaluation modeling } \\
\text { method based on } \\
\text { multidimensional data } \\
\text { mining }\end{array}$ \\
\hline 10 & 1.58 & 1.74 & 0.84 \\
20 & 1.24 & 2.12 & 0.97 \\
30 & 0.98 & 1.56 & 0.65 \\
40 & 0.77 & 1.25 & 0.74 \\
50 & 1.68 & 1.39 & 0.58 \\
\hline
\end{tabular}




\begin{tabular}{cccc}
\hline 60 & 1.54 & 2.01 & 0.47 \\
70 & 1.57 & 2.35 & 0.68 \\
80 & 1.84 & 2.44 & 0.63 \\
90 & 1.62 & 1.98 & 0.85 \\
100 & 1.37 & 1.47 & 0.64 \\
The average & 1.42 & 1.83 & 0.71 \\
\hline
\end{tabular}

Analysis of the data in Table 3, the evaluation method based on the pressure - state - response model of the evaluation takes the average of $1.42 \mathrm{~s}$, based on the center of the triangle whitenization weight function of the evaluation method of evaluation takes the average of $1.83 \mathrm{~s}$, government debt risk evaluation based on multidimensional data mining modeling methods of evaluation takes the average of $0.71 \mathrm{~s}$, compared with the two methods, the proposed method has the shortest time and higher efficiency for the comprehensive evaluation of government debt risk, which can achieve the goal of rapid comprehensive evaluation of government debt risk.

\section{Conclusion}

With the rapid development of social economy, the local governments gradually build and perfect the mechanism of debt financing, but once the government facing debt risk is too big, will inevitably hurt the local economy and people, so in order to effectively solve these problems, need to research an effective government debt risk evaluation method. But the current government debt risk evaluation method of low computational accuracy evaluation indexes, the evaluation of the relative error is higher and the evaluation takes longer, so this paper in order to solve the problems existing in the current method as the research target, this paper proposes a new government debt risk comprehensive evaluation on the basis of multidimensional data mining modeling methods. The experimental results show that the data mining accuracy of this method is always above $91 \%$, the relative error rate of evaluation is always below $3.4 \%$, and the average evaluation time is $0.71 \mathrm{~s}$, which can realize the rapid and accurate evaluation of government debt risks. It is hoped that the relevant parts in the future will take this study as the basis to ensure the safety of government funds and promote the steady and rapid development of social economy by clearly dividing the boundary between state-owned enterprises and local government debts, monitoring debt risks, increasing government financing guarantee funds and other ways.

\section{Declarations}

Conflicts of interest/Competing interests: Not applicable

Availability of data and material: Not applicable

Code availability: Not applicable

Authors' contributions: Li ChaoYing - Data Collection, Supervision, Resources; Wu Xiang Da - Analysis,

Software, Manuscript- Initial editing; Zhao En Hui - Concept and design, Results interpretation, ManuscriptFinal editing. Li ChaoYing, Wu Xiang Da, Zhao En Hui have read and approved the manuscript.

\section{Compliance with Ethical Standards}

Disclosure of potential conflicts of interest: Not applicable

Research involving Human Participants and/or Animals: Not applicable

Informed consent: Not applicable

\section{References}

[1]Xu Z, Zhu F, Fan W. Establishment of early warning index of local government debt risk-taking Hubei province of China as an example[J]. IOP Conference Series Materials Science and Engineering, 2020, 768(1):052099-052112.

[2]Zhang R, Shi R, Li C. Local government debt risk measurement and early warning structural system building-Research 
Based on KMV and TOPSIS Models[J]. E3S Web of Conferences, 2021, 235(1):02039-02048.

[3]Mcab C, Ttnd E, Sr E. Persistent Government Debt and Aggregate Risk Distribution[J]. Journal of Financial Economics, 2021, 140(2):347-367.

[4]Xie B P, Zhu D L, Chen Y. Risk assessment of local government debt based on "pressure-state-response" model[J]. China Development, 2019, 19(4):14-21.

[5]Hu C L. Research on risk assessment of local government debt -- grey assessment model based on central point triangular whitening weight function [J]. Financial Supervision, 2020, 46(4):69-73.

[6]Zhu Wenwei. Study on the Debt Risk Assessment of Local Government under the Dual Goal of Steady Economic Growth and Risk Prevention[J]. Contemporary Economy \& Management, 2019, 41(2):68-76.

[7]Loey M, Jasim M W, El-Bakry H M, et al. Breast and Colon Cancer Classification from Gene Expression Profiles Using Data Mining Techniques[J]. Symmetry, 2020, 12(3):408-415.

[8]Mengash H A. Using Data Mining Techniques to Predict Student Performance to Support Decision Making in University Admission Systems[J]. IEEE Access, 2020, 8(1):55462-55470.

[9]Wu D, Zhang Y. Design of Remote Monitoring System of Harvester_—Based on Parallel Algorithms of Cloud Platform Data Mining[J]. Journal of Agricultural Mechanization Research, 2020, 42(6):235-239.

[10]Liu J. Improvements on Sample Selection in Data Mining Based on Audit Opinions and Regulatory Measures[J]. Journal of Nanjing University of Science and Technology(Social Sciences Edition), 2020, 33(1):42-47.

[11]Moayedi H, Abdullahi M M, Nguyen H, et al. Comparison of dragonfly algorithm and Harris hawks optimization evolutionary data mining techniques for the assessment of bearing capacity of footings over two-layer foundation soils[J]. Engineering with Computers, 2021, 37(1):437-447.

[12] D Kumalasari, Putra A, Gaffar A. Speech classification using combination virtual center of gravity and k-means clustering based on audio feature extraction[J]. Jurnal Informatika, 2020, 14(2):85-96.

[13]Liu H, Chen L, Mu L, et al. A Recognition Method of Kiwifruit Flowers Based on K-means Clustering[J]. Journal of Agricultural Mechanization Research, 2020, 42(2)L22-26.

[14]Holilah D, Bustamam A, Sarwinda D. Detection of Alzheimer's disease with segmentation approach using K-Means Clustering and Watershed Method of MRI image[J]. Journal of Physics Conference Series, 2021, 1725(1):012009-012016.

[15]Huang S W, Wang F, Cao Q W, et al. Research on Fast Multi-objects Feature Point Matching Based on Cluster Algorithm[J]. Computer Simulation, 2019, 36(2):211-214+376. 